\title{
Importance of wasp and honey bee recombinant allergens in the diagnosis of hymenoptera venom anaphylaxis
}

\author{
Radosław Gawlik', Magdalena Marcak², Andrzej Bożek² \\ ${ }^{1}$ Chair and Department of Internal Diseases, Allergology, Clinical Immunology \\ ${ }^{2}$ Clinical Department of Internal Diseases, Dermatology and Allergology in Zabrze, \\ Medical University of Silesia in Katowice, Poland
}

Abstract:

Background: Hymenoptera venom-allergic patients frequently present multiple sensitisations.

Objectives: To define the allergic profile by components in Hymenoptera venom allergic patients. To study the usefulness of specific lgE to components in cases of negative results of slgE to the venoms.

Patients $(n=86)$ with a diagnosis of allergic systemic reaction following honey bee or wasp stings were included in the study. Skin prick tests (SPT) and intradermal reaction were performed.

slgE to complete extracts of honey bee (Apis mellifera), Vespula (Vespula spp.), venom and recombinant allergens rApi m1, rApi $\mathrm{m} 5$; rVes v5 and rVes v1 5 were analysed by ImmunoCAP (Phadia). slgE concentration higher than $0.35 \mathrm{kU} / \mathrm{l}$ were considered positive.

Results: 86 patients ( 40 male, 46 female) were included. No slgE to Honey bee/Vespula was detected in 15 patients. In 7 of them only component diagnosis (Api $\mathrm{m} 1$ and Ves v5) confirmed hymenoptera allergy. Positive correlation between severity of anaphylactic reaction and slgE to rVes v1 concentration was observed $(r=0,85 ; p<0.05)$ and slgE to rApi $m 1(r=0.87 ; p<0.05)$. None such correlation was observed with specific lgE to other venom components.

Conclusions: Components analysis can be useful to make diagnosis more accurate. Determination of slgE towards phospholipase A2 (Apis mellifera) and phospholipase A1 (Vespula spp.) may reflects severity of allergic reaction after hymenoptera sting.

Key words: hymenoptera venom allergy, diagnosis

A naphylaxis is a rapidly developing and life-threatening hypersensitivity reaction of the body in response to a triggering allergen and is the most dangerous atopic disease. Anaphylaxis represents a significant health care burden. The most common causes of anaphylaxis are foods, medications and insects. Anaphylaxis occurs in approximately $1-3 \%$ of people annually [1]. In the US, it occurs at least once in a lifetime for $2 \%$ of the population [2]. The incidence of anaphylactic reactions is increasing, mostly in young people and women. Different studies have shown that the incidence of anaphylaxis is much higher than was previously estimated $[3,4]$. Allergy to hymenoptera venom is an important medical problem. Hymenoptera anaphylaxis is one of the leading causes of severe allergic reactions and can be fatal. The incidence of an allergic reaction after insect stings ranges from $0.15 \%$ to $0.8 \%$ in children and from $0.3 \%$ to $8.9 \%$ in adults [5]. The rate of reported systemic sting reactions (SSRs) and ranges from $0.3 \%$ to $7.5 \%$ in latest European epidemiological studies [6]. Stinging of a sensitized subject by a hymenoptera insect is a life-threatening issue [1]. The severity of anaphylaxis depends on the individual sensitivity of the patient, concomitant diseases, and genetic and environmental factors. Thus far, no parameters have been identified that can predict who will be 
at risk for an allergic reaction after a sting or what the severity will be [5]. Knowledge of the composition of venoms and structure of allergens is a prerequisite for the accurate diagnosis and treatment of insect venom allergy. Hymenoptera venoms are a complex mixture of biogenic amines, peptides, toxins, enzymes, and low-weight proteins. Among different Hymenoptera venoms, honey bee venom (HBV) is currently the best characterized. A total of about 105 compounds among different Hymenoptera venoms were described and listed in the WHO/IUIS Allergen Nomenclature official database [7]. Actually, out of these compounds, 5 allergens have been identified in HBV. Phospholipase A2 (Api m1), hyaluronidase (Api m2), acid phosphatase (Api m3), dipeptidyl peptidase IV (Api m5), and/or icarapin (Api m10) are those allergens of high abundance in HBV and specific IgE against those compounds have been identified in $94.4 \%$ of allergic patients. In yellow jacket (V. vulgaris) venom, phospholipase A 1 (Ves v1) and antigen 5 (Ves v5) have been identified as major allergens.

The only, very effective causative treatment is allergen immunotherapy, venom immunotherapy with a protection rate ranging from $75 \%$ to $98 \%$. Their effectiveness, however, depends on precise diagnosis and appropriate selection of the vaccine. One of the diagnostic problems are negative skin tests and no detectable venom specific IgE in the serum of anaphylactic patients.

Evaluation of serum allergen specific immunoglobulin E (sIgE) to hymenoptera venoms in relation to usefulness of specific IgE to their components was an objective of the study. We want also to study the usefulness of specific IgE to components in cases of negative results of $\mathrm{sIgE}$ to the venoms.

\section{Materials and methods}

We included into the study 86 patients (40 male, 46 female) with anaphylactic reaction to wasp or honey bee sting.

Diagnosis was made on the basis of medical history. Diagnosis of wasp or honey bee (Vespula spp., Apis mellifera) venom allergy was made on the basis of medical history of anaphylactic sting reactions, positive skin test responses, and/or detection of specific IgE to wasp or honey bee venom.

Anaphylaxis was diagnosed according to the clinical definition presented by the World Allergy Organization [8], which required documented symptoms or signs of the involvement of 2 or more organ systems, including the skin, respiratory (dyspnoea), gastrointes- tinal and cardiovascular systems (syncope, loss of consciousness). The severity of the anaphylactic reaction was estimated according to Muller's scale.

In all patients skin prick tests and stepwise incremental venom intradermal skin tests with wasp and honey bee venom extracts were performed (Pharmalgen, ALK, Denmark).

The following sIgE were determined in the serum of investigated patients: sIgE to complete extracts of honey bee (Apis mellifera), Vespula (Vespula spp.) venom and recombinant allergens of honey bee rApi m1 (fospholipase A2), rApi m5 (allergen C) and wasp rVes v1 (fospholipase A1), rVes v5 (antygen 5), MUXF3 CCD bromelin and analysed by Immuno CAP 250 (Phadia Gmbh, Freiburg, Germany). sIgE values greater than $0.35 \mathrm{kU} / 1$ were considered positive.

The data were analyzed using Statistica software. Comparison of continuous variables that were not normally distributed was performed using the Mann-Whitney U test and were expressed as medians with $95 \%$ CI. The data from normally distributed continuous variables were analysed with ANOVA and expressed as the means with SD.

\section{Results}

Table 1. Characteristics of the patients.

\begin{tabular}{|l|l|l|l|}
\hline Patients allergic to & \multicolumn{1}{|c|}{ Age (years) } & \multicolumn{1}{c|}{$\begin{array}{c}\text { Severity of } \\
\text { anaphylaxis }\end{array}$} & \multicolumn{1}{|c|}{ Gender } \\
\hline Wasp venom & $21-68 ; 49.4 \pm 7.2$ & 3.2 & $18 \mathrm{M} ; 25 \mathrm{~F}$ \\
\hline Honey bee venom & $20-66 ; 471.3 \pm 6.4$ & 3.6 & $22 \mathrm{M} ; 21 \mathrm{~F}$ \\
\hline
\end{tabular}

There was observed positive correlation between sIgE to r Ves v1 (i 209) and severity of anaphylaxis acc to Muller after wasp sting $(\mathrm{r}=0.85$; $\mathrm{p}<$ 0.05) (fig. 1).

No such correlation was registered with another investigated wasp venom component, antigen 5. - rVes v5 $(\mathrm{r}=0.38 ; \mathrm{p}<0.05)$.

Correlation of sIgE to complete extract of wasp and severity of anaphylaxis acc to Muller after wasp sting was weaker, but still positive $(r=0.67 ; p<0.05)$.

Similar result was observed with $\mathrm{sIgE}$ to honey bee and anaphylaxis $(\mathrm{r}=0.71 ; \mathrm{p}<0.05)$.

There was observed positive correlation between $\operatorname{sIgE}$ to rApi $\mathrm{ml}$ and severity of anaphylaxis acc to Muller after honey bee sting $(r=0.64 ; p<0.05)$.

The study found that concentration of $\operatorname{sigE}$ to whole honey bee extract correlate in different ways with $\mathrm{sIgE}$ to the compund of the venom being positive 
Figure 1. Correlation between sIgE to wasp venom component $r V e s v 1$ and severity of anaphylactic reaction $(r=0.85 ; p<0.05)$.

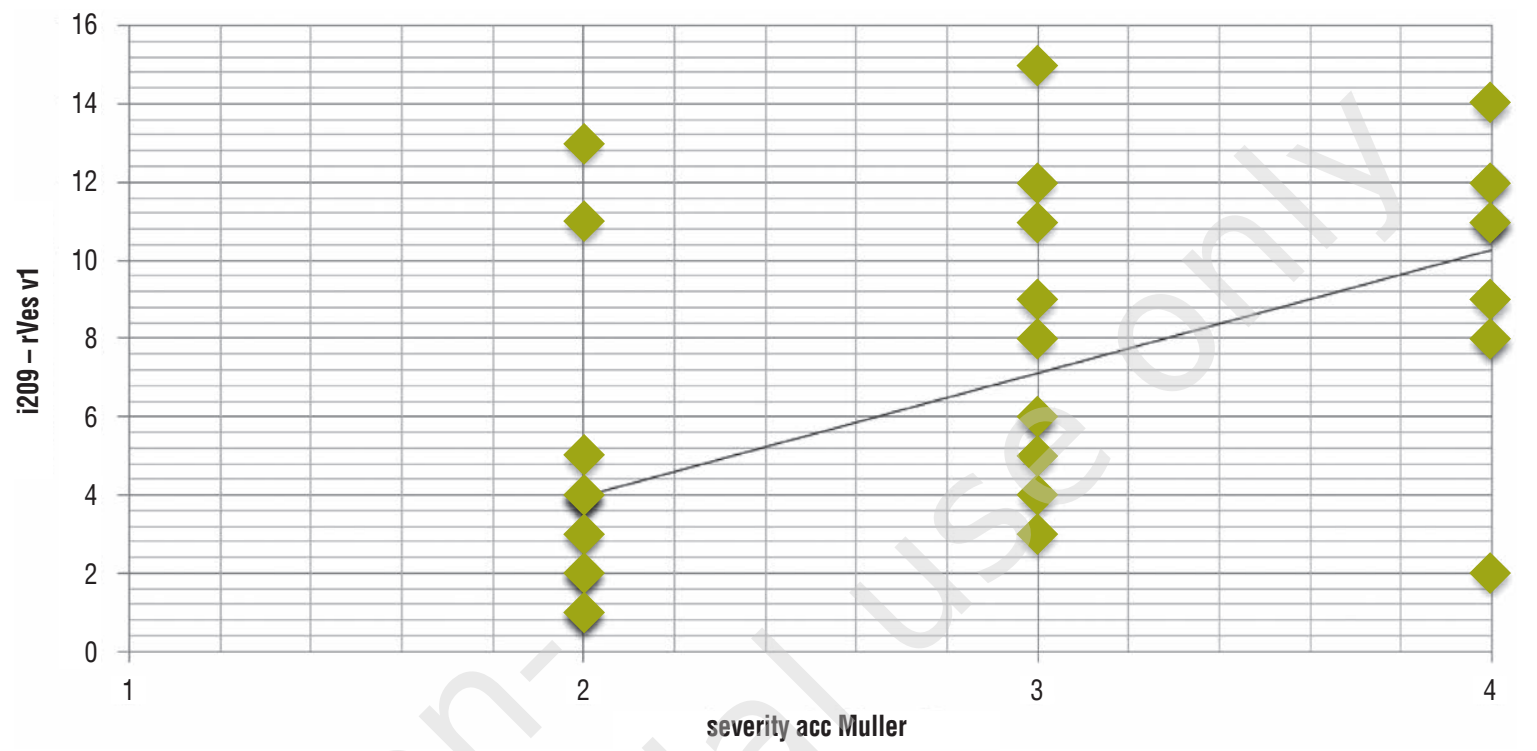

with $\mathrm{sIgE}$ to $\mathrm{rApi} \mathrm{m} 1 ; \mathrm{r}=0.87 ; \mathrm{p}<0.05)$, and without statistical correspondence with rApi m10.

Analysis of the relationship between concentrations of sIgE to wasp whole venom (Vespula spp.) and individual compounds of the venom showed positive correlation only with $\mathrm{rVes} v 1 ; \mathrm{r}=0,42 ; \mathrm{p}<0.05$ demonstrating its absence with rVes v5.

Among 15 patients with negative results of sIgE to complete both venoms, 7 subjects revealed specific IgE to their components (Api m1, Ves v1; $n=3 / 4$ ) and helped to confirm suspected diagnosis of venom sensitivity. sIgE to Vespula (Phadia) was negative in eight patients but three of them showed positive sIgE to rVes v5 (Phadia) but only two patients to rVes v1. sIgE to complete extracts of honey bee (Apis mellifera) (Phadia) was negative in 7 patients but 4 of them showed positive sIgE to rApi $\mathrm{m} 1$ but only in 1 to rApi m5 (Phadia).

\section{Discussion}

In patients who have suffered from a systemic reaction after a Hymenoptera sting, the severity of the next reaction cannot be predicted from the previous reaction, as the amount of venom can differ [6]. In addition, cofactors, such as intake of drugs ( $\beta$-blockers and ACE inhibitors), illness, stress, or alcohol, can increase the severity of allergic symptom. Observed gaps in the diagnosis of insect venom anaphylactic patients were previously caused by imperfect venom extracts lacking a few important allergens (e.g. icarapin, vitellogenins). This imperfection makes it difficult to make a decision to start venom immunotherapy, leaving these patients without adequate and effective therapy. A small group of patients reporting systemic reactions to insect stings had no detectable venom specific IgE in their serum and were "negative" at skin testing [9]. This could be due to insufficient sensitivity of tests, or to a long interval from the sting-induced reaction to testing with spontaneous decline in venom specific $\operatorname{IgE}[10]$. The failure to detect venom specific IgE provides no guarantee that the clinical reactivity has waned.

Use of component-resolved diagnosis enables diagnosed venom allergy in 7 patients with so far not proved sensitization.

The current treatment for insect venom allergy includes advice to minimise exposure to further stings, prescription of self-administered adrenaline, and venom immunotherapy (VIT) in selected people.

However, positive skin tests or serologic results obtained with conventional venom extracts do not always reflect a primary sensitization. Crude venom extracts used conventionally for diagnosing Hymenoptera allergy not always reflect real sensitization and sometimes is misdiagnosed because of allergen cross-reactivity.

For the cross-reactivity are in $50 \%$ responsible anti-N-Glycan cross-reactive carbohydrate determinant (CCD) IgE antibodies [11-13].

Majority of insect venom allergic patients $(66 \%)$ demonstrates hypersensitivity to one species specific recombinant venom allergen [14]. In our study only few patients were double sensitive. Limitation of presented study was ability to determine selected anti- 
bodies from among complete panel of wasp and honey bee compounds e.g. Api m1, 2, 3, 5, 10.

Currently, there is no pattern of sensitization of allergens known which can predict safety or efficacy of a specific immunotherapy. We observed that severity of anaphylaxis correlated positive with serum concentration of sIgE to $r$ Ves v1 in wasp allergic patients and sIgE to rApi $\mathrm{m} 1$ in honey bee allergic ones. Authors of a small study cohort with 31 patients treating patients either with native or purified aqueous $\mathrm{HBV}$ extracts according to IgE levels of Api $\mathrm{m} 4<$ or $\geq 0.98 \mathrm{kU} \mathrm{A} / \mathrm{L}$ respectively, reported a higher rate of side effects and less success after insect sting challenge in the group of patients with higher IgE to Api m4 [15].

Sensitivity to allergen components of honey bee venom and specially wasp ones differ according to dominating in this geographical region species and type of diagnostic venom extract used.

There were reported controversial attempts adding recombinant allergens to improve the detection of Hymenoptera venom allergic patients in addition to spiking wasp venom extract with Ves v5 [16].

Phospholipase A2 (Api m1) has been considered the most important and potent allergen of bee venom. We observed its good correlation with sIgE to whole body extract. In other studies with the bee venom compound-based diagnosis, it has been observed that use of allergen Api $\mathrm{m} 1$ has different sensitivity according to the technique and sources used [17]. Component-resolved diagnosis based on the use of well-defined, properly characterized and purified natural and recombinant allergens constitutes a new approach in the diagnosis of venom allergy.

We consider CRD shall be used as a complementary diagnostic tool, linked to patient's history, skin-prick testing, and specific-IgE determination, rather than a first-line choice. It may allow identification of biomarkers, which can be used for risk stratification in VIT and enable the selection of appropriate preparations for VIT according to personalized sensitization profile.

The diagnostic gap of previously undetected Hymenoptera allergy has been decreased through introduction of recombinant allergens. Knowledge of analogies in interspecies proteins and cross-reactive carbohydrate determinants is necessary to distinguish relevant from irrelevant sensitizations. The obtained information contributes to an increased diagnostic precision in HVA as we also proved in the study but still few questions remained unanswered. The benefit of the commercially component-resolved diagnosis in the present form is questionable, due to the insufficient sensitivity [18]. This diagnostic method is still challenging one.

We consider CRD shall be used as a complementary diagnostic tool, linked to patient's history, skin-prick testing, and specific-IgE determination, rather than a first-line choice.

\section{References}

1. Simons FER, Ardusso LRF, Bilo MB et al. International consensus on (ICON) anaphylaxis. WAOJ 2014, 7: 9.

2. Wood RA, Camargo Jr CA, Lieberman P et al. Anaphylaxis in America: The prevalence and characteristics of anaphylaxis in the United States. J Allergy Clin Immunol 2014, 133: 461-467 .

3. Mullins RJ, Dear KBG, Tang MLK. Time trends in Australian hospital anaphylaxis admissions in 1998-1999 to 2011-2012. J Allergy Clin Immunol 2015, 136: 367-375.

4. Turner PJ, Gowland MH, Sharma V et al. Increase in anaphylaxis-related hospitalizations but no increase in fatalities: An analysis of United Kingdom national anaphylaxis data, 1992-2012. J Allergy Clin Immunol 2015, 135: 956-963.

5. Stewart $A G, E w a n P W$. The incidence, aetiology and management of anaphylaxis presenting to an accident and emergency department. QJM 1996, 89: 859-864.

6. Bilo $M B$, Bonifazi $F$. The natural history and epidemiology of insect venom allergy: clinical implications. Clin Exp Allergy 2009, 39: 1467-1476.

7. WHO/IUIS Allergen Nomenclature-subcommettee database [online: www.alergen.org].

8. Simons FE, Ardusso LRF, Bilo MB et al. 2015 update of the evidence base: World Allergy Organization anaphylaxis guidelines. World Allergy Organ J 2015, 8: 32. DOI: 10.1186/ s40413-015-0080-1.

9. Golden DBK, Kagey-Sobotka A, Norman PS et al. Insect sting allergy with negative venom skin test responses. J Allergy Clin Immunol 2001, 107: 897-901.

10. Kontou-Fili K. Patients with negative skin tests. Curr Opin Allergy Clin Immunol 2002, 2: 353-357.

11. Jappe U, Raulf-Heimsoth M, Hoffmann $M$ et al. In vitro Hymenoptera venom allergy diagnosis: improved by screening for cross-reactive carbohydrate determinants and reciprocal inhibition. Allergy 2006, 61: 1220-1229.

12. Jakob T, Kohler J, Blank S et al. Comparable IgE reactivity to natural and recombinant Api $m 1$ in cross-reactive carbohydrate determinant-negative patients with bee venom allergy. J Allergy Clin Immunol 2012, 130: 276-278.

13. Seismann H, Blank S, Braren I et al. Dissecting cross-reactivity in hymenoptera venom allergy by circumvention of alpha-1,3-core fucosylation. Mol Immunol 2010, 47: 799-808.

14. Muller $U$, Johansen $N$, Petersen A et al. Hymenoptera venom allergy: analysis of double positivity to honey bee and Vespu- 
la venom by estimation of IgE antibodies to species specific major allergens Api $m 1$ and Ves v. Allergy 2009, 64: 543-548.

15. Ruiz B, Serrano P, Moreno C. IgE-Api $m 4$ is useful for identifying a particular phenotype of bee venom allergy. J Investig Allergol Clin Immunol 2016, 26: 355-361.

16. Vos B, Koehler J, Euller SM et al. Spiking venom with rVes $v 5$ improves sensitivity of IgE detection in patients with allergy to Vespula venom. J Allergy Clin Immunol 2012, 131: 1225-1227.

17. Sturm GJ, Hemmer W, Hawranek $T$ et al. Detection of IgE to recombinant Api $m 1$ and $r$ Ves $v 5$ is valuable but not sufficient to distinguish bee from wasp venom allergy. J Allergy Clin Immunol 2011, 128: 247-248.

18. Arzt L, Bokanovic D, Schrautzer C et al. Questionable diagnostic benefit of the commercially available panel of bee venom components. Allergy 2017, 72(9): 1419-1422.
ORCID

Radoslaw Gawlik: 0000-0002-8537-9165

Authors' contributions: Gawlik R: study project, data collection, preparation of the manuscript; Marcak M: data collection; Bozek A: results analysis, preparation of the manuscript. Conflict of interests: The authors declare that they have no competing interests.

Financial support: The study was financed by a grant from the Silesian Medical University: KNW-1 - 068/N/5/0.

\section{Corresponding author:}

prof. Radosław Gawlik, MD, PhD

Chair and Department of Internal Diseases,

Allergology, Clinical Immunology;

Medical University of Silesia Katowice

40-514 Katowice, Ceglana 35

e-mail: radwags@poczta.onet.pl

C Medical Education. For private and non-commercial use only. Downloaded from https://www.journalsmededu.pl/index.php/alergoprofil: 26.04.2023; 04:38,37 